\title{
Efficacy of supplementation with methylcobalamin and cyancobalamin in maintaining the level of serum holotranscobalamin in a group of plant-based diet (vegan) adults
}

\author{
CORINA-AURELIA ZUGRAVU ${ }^{1 *}$, ADRIANA MACRI $^{2 *}$, NASTASIA BELC $^{2 *}$ and ROXANA BOHILTEA ${ }^{3,4}$ \\ ${ }^{1}$ Department of Hygiene and Ecology, Faculty of Nursing and Midwifery, 'Carol Davila' University of \\ Medicine and Pharmacy, 020021 Bucharest; ${ }^{2}$ Department of Biotechnology, National Institute of Research \\ and Development for Food Bioresources, 014192 Bucharest; ${ }^{3}$ Department of Obstetrics and Gynecology, \\ 'Carol Davila' University of Medicine and Pharmacy, 020021 Bucharest; ${ }^{4}$ Department of Obstetrics \\ and Gynecology, University Emergency Hospital Bucharest, 050098 Bucharest, Romania
}

Received April 30, 2021; Accepted May 31, 2021

DOI: $10.3892 / \mathrm{etm} .2021 .10425$

\begin{abstract}
Plant-based nutrition has become extremely popular in the contemporary era. Its positive effects are sustained by many studies, but one of its risks is that it is almost completely devoid of vitamin B12. In the present study, we analyzed the effects of two types of vitamin B12 supplements, cyancobalamin and methylcobalamin on the level of active serum vitamin (holotranscobalamin) in a group of Romanian individuals $(n=42)$ following a (vegan) plant-based diet. The results revealed that cyancobalamin gives better results in maintaining B12, as quantified by the holotranscobalamin value $($ median $=150 \mathrm{pcg} / \mathrm{l})$ when compared with methylcobalamin (median=78.5 pcg/l). The frequency of administration, regardless of the quantity in one dose, is another important factor in maintaining the holotranscobalamin level within suitable limits. More frequent intakes give more optimal results. Vegans trying to supplement with alternative products (algae, kombucha, other fermented products), had the lowest levels of holotranscobalamin, always bellow the recommended level of $35 \mathrm{pcg} / \mathrm{l}$ (median=29 pcg/l). Vegans must be educated on B12 supplementation, about the pharmaceutical forms on the
\end{abstract}

Correspondence to: Dr Corina-Aurelia Zugravu, Department of Hygiene and Ecology, Faculty of Nursing and Midwifery, 'Carol Davila' University of Medicine and Pharmacy, 37 Dionisie Lupu Street, 020021 Bucharest, Romania

E-mail: corina.zugravu@umfcd.ro

Dr Roxana Bohiltea, Department of Obstetrics and Gynecology, 'Carol Davila' University of Medicine and Pharmacy, 37 Dionisie Lupu Street, 020021 Bucharest, Romania

E-mail: r.bohiltea@yahoo.com

*Joint senior authorship

Key words: holotranscobalamin, plant-based diet, cyancobalamin, methylcobalamin, vegan, vitamin B12 supplements market and their performances and on choosing the optimal plan in order to avoid the onset of B12 deficiency.

\section{Introduction}

Vitamin B12 is the latest vitamin discovered in the first half of the 20th century, and the emergence of B12 supplements has been a key moment in addressing pathological conditions such as pernicious anemia. Not only in various diseases have they proven to be valuable, but also in dietary contexts when the intake is minimal (1). Bacteria achieve the synthesis of vitamin B12 in the mammalian food chain. By means of water or food, the vitamin reaches the meat and organs of farm animals and from here, through a relatively laborious process of absorption, humans can utilize it. Because the maximum amounts of vitamin B12 are found in the viscera (liver) of mammals and fish and in the meat of some fish products not very abundant in the diet of modern man, the intake is quite small (1). However, the metabolism of vitamin B12 is an economical one, the daily losses being minimal. That is why deficiency in healthy adults rarely occurs. In addition, despite the fact that it is a water-soluble vitamin, the body ends up making small liver reserves (2), related to food intake. Primitive man probably survived, even in the absence of abundant food sources, consuming various products with a rich bacterial presence.

One example of a diet in which the intake of B12 is inadequate is the plant-based diet, a diet currently adopted by more and more individuals, sometimes named 'vegans', even though this term has a wider significance. It should be noted that often pregnant women, the elderly or children, age groups prone to different deficiencies, are eating relevant to this model, thus the interest given to B12 insurance is even more justified (3). There are no reliable and constant sources of vitamin B12 in the plant world to date, some studies highlighting the presence of inconsistent amounts in niche products $(4,5)$. Of course, processed plant foods can be fortified directly (e.g., vegetable milk) or by cultivation on a substrate enriched with B12 (some brands of nutritional yeast). Yet, vitamin B12 deficiency, even 
if it becomes symptomatic after many years of absence from the diet, has disastrous consequences, and it is frequently misdiagnosed; therefore, the general rule is to recommend supplementation (6).

There are different forms of vitamin B12 on the food supplement market. The most common forms are cyancobalamin (cyanCbl) and methylcobalamin (methylCbl). MethylCbl is recommended by manufacturers with the argument that it is the ready-to-use form of the vitamin, while cyanCbl must be activated before it can be used metabolically. This ignores the fact that upon absorption, methylCbl is dissolved, and the methyl group removed. In order to obtain usable methylCbl, the active coenzyme, the body needs to re-form it endogenously. As such, there is no metabolic superiority of methylCbl supplements (7). There are no differences between cyanCbl and methylCbl supplements in terms of the effort made by the healthy body to use them. Various laboratory tests have been proposed to measure the adequacy of vitamin B12 intake: Serum B12 levels, holotranscobalamin (holoTC), methylmalonic acid (MMA) or homocysteine levels. Unfortunately, these tests are not commonly performed and are expensive. Recently, the measurement of holoTC has been imposed as a precise first step in measuring the adequacy of B12 intake, considering that several types of subsequent tests will provide a better assessment of the individual's nutritional status (8).

The present study aimed to analyze the level of active serum cobalamine (holoTC) depending on the type of cobalamin used for supplementation, in a group of people with a plant-based diet, to highlight the product with the best results.

\section{Subjects and methods}

Within the CORE project, one of the included modules was of a group of individuals with plant-based nutrition (vegans) $(n=150)$. The choice of participants was made by a procedure similar to that described in another article (9). The test selected as providing the best status data in B12 was holoTC. Profile studies have shown that this marker is specific for highlighting B12 deficiency, performing much better than determining total B12 in serum, especially in cases where the deficiency is marginal (10-12). In addition, its determination is more acceptable in terms of cost compared to methylmalonic acid (MMA).

After completing the data collection within the CORE project, vegans were contacted by telephone and asked about the possibility of having their vitamin B12 assessed in the previous 6 months at an authorized laboratory. Out of the total of 150 people, 60 individuals who agreed to participate in the present investigation responded positively in the first phase. None of the respondents reported a chronic condition diagnosed by the doctor (as found from the answers to the initial questionnaire, applied in the CORE study).

The study continued by administering a new questionnaire using a computer-assisted telephone interviewing (CATI) method. The telephone questionnaire included general questions concerning: i) demographics (age, sex and educational level of the respondents); ii) regarding possible current pathology; iii) to confirm the status of the participant as a consumer of an exclusively plant-based diet (vegans) (to note, in the previous stages of the study, it was found that many individuals declared themselves vegans but in fact also consumed products of animal origin); iv) regarding the duration of the adoption of this diet; v) regarding the supplementation with vitamin B12 (duration of supplementation, form administered, pharmaceutical form, average daily amount of vitamin); vi) regarding the consumption of fortified foods with B12 (vegetable drinks, vegetable cheese substitutes, meat substitutes); and vii) the value obtained from the laboratory analysis. In addition, the respondents were asked to send via E-mail a scanned copy of the analysis bulletin. After this first phase, 8 individuals were excluded, in which case the analysis report did not confirm the performance of any determination of vitamin B12 or associated metabolites. In addition, 10 individuals were excluded as the test did not refer to holoTC but to one other parameter. The final group consisted of 42 individuals, 25 men and 17 women, with an average age of 34 years (maximum age, 65 years; minimum age, 21 years).

Statistics. The results obtained in the questionnaire and the data of the laboratory tests were statistically processed using SPSS 12.0 software (SPSS, Inc.), applying descriptive, t-tests, correlation and linear regression tests, with the holoTC value as a dependent variable.

\section{Results}

Depending on the type of vitamin B12 supplemented, the group was divided into 3 categories: Vegans who supplement with cyanCbl (group 1), vegans who supplement with methylCbl (group 2) and vegans who supplement with products identified as 'natural' and about which they read/think are rich in B12 (algae, kombucha, borscht, yeast) (group 3). Group 1 consisted of 21 members, group 2 included 14 participants and group 3, 7. None of the respondents reported the habitual consumption of products fortified with vitamin B12. This aspect can be explained by the fact that these foods are not commonly available on the Romanian market where vegetable products are usually unfortified, as well as the unjustified fear of consuming fortified products as an undesirable way of eating processed food with 'artificial' vitamins.

The holoTC values in the working groups are presented in Fig. 1. As provided by their results, the laboratories used the radioimmunoassay method to evaluate holoTC, and considered that a value under $35 \mathrm{pmol} / \mathrm{l}$ signals the start of a depletion of B12 reserves, thus indicating the necessity of further analysis with an MMA measurement. It was noted that the median was higher for group 1, but still the dispersion range of values in groups 1 and 2 were quite high, the determinant factors being most probably the daily dose, the intake frequency and the individual metabolic peculiarities. None of the parameters were adequate for group 3; for group 2, however, there were just 2 individuals below the limit (less than 35 pmol/l) (Table I).

The results of the t-test showed significant differences between all 3 groups in terms of holoTC value. Group 3 was significantly different from groups $1(\mathrm{P}=0.001)$ and 2 $(\mathrm{P}=0.0012)$, but also between groups 1 and 2 there was a statistically significant difference $(\mathrm{P}=0.001)$.

The average daily amount supplemented in groups 1 and 2 varied between 25 and 1,000 mcg. For group 3 such a value could not be calculated. The average amount per intake in group 1 was $582 \mathrm{mcg}(\mathrm{SD}=505.42)$ and that in group 2, $988 \mathrm{mcg}(\mathrm{SD}=734)$, with the difference not achieving statistical 
Table I. HoloTC values in the 3 vegan groups (pmol/l).

\begin{tabular}{lcccc}
\hline HoloTC values & Group 1 & Group 2 & Group 3 & P-value \\
\hline Mean value & 133.7 & 81.5 & 27 & 0.001 \\
Max & 185 & 150 & 32 & 0.002 \\
Min & 54 & 28 & 21 & 0.040 \\
Median & 150 & 78.5 & 29 & 0.030 \\
\hline
\end{tabular}

HoloTC, holotranscobalamin. Groups: The subjects were divided into 3 categories: Vegans who supplement with cyanCbl (group 1), vegans who supplement with methylCbl (group 2) and vegans who supplement with products identified as 'natural' and about which they read/think are rich in B12 (algae, kombucha, borscht, yeast) (group 3).

Table II. Duration of B12 supplementation and adherence to a vegan diet (years).

\begin{tabular}{|c|c|c|c|c|}
\hline & Mean value & Max & Min & Median \\
\hline Duration of veganism & 5.7 & 18 & 1 & 5 \\
\hline $\begin{array}{l}\text { Duration of B12 } \\
\text { supplementation }\end{array}$ & 3.57 & 17 & 1 & 2 \\
\hline
\end{tabular}

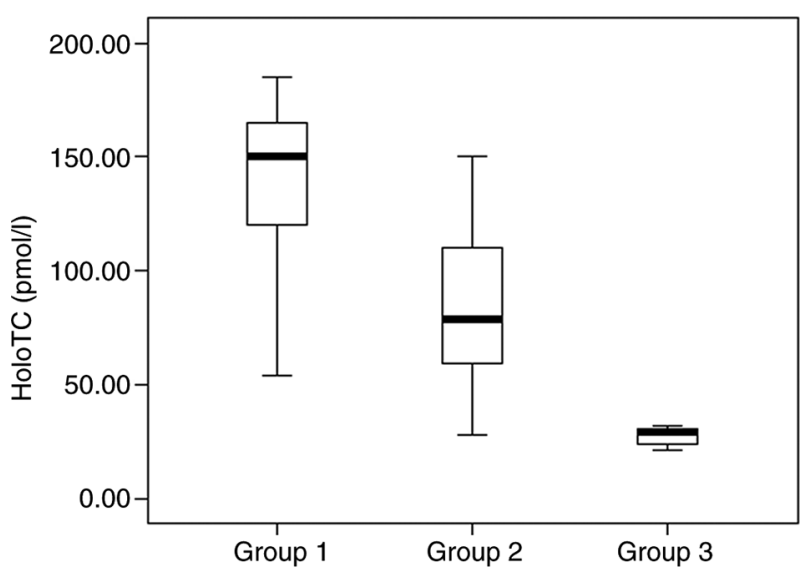

Figure 1. Values of holoTC (pmol/l) in the 3 groups. Groups: Subjects were divided into 3 categories: Vegans who supplement with cyanCbl (group 1), vegans who supplement with methylCbl (group 2) and vegans who supplement with products identified as 'natural' and about which they read/think are rich in B12 (algae, kombucha, borscht, yeast) (group 3).

significance. We noted that the methylCbl one-time dose was higher than the cyanCbl one, but the dispersion of the values in group 2 were extremely large.

There was a difference in the mean and median values in terms of the duration of the vegan diet and the duration of the supplementation for all 3 groups combined, which showed that many vegans do not supplement as soon as they give up the consumption of animal products (Table II).

Regarding the pattern of supplementation during a week, 13 individuals supplemented once, 18 twice, 1 thrice and 10 individuals took supplements every day. The pattern was different in groups 1 and 2, with more individuals supplementing daily in the first group, than in the second, but the difference was not statistically significant $(\mathrm{P}=0.054)$. For group 3 (not included in Table III), supplementation appeared to be mostly daily. We analyzed the association between the level of holoTC and the pattern of administration of the supplements (Fig. 2A) and values dependent on both pattern and the type of supplement (Fig. 2B). Highest median values were obtained with more frequent intake of the supplements (Fig. 2A) and the frequency appeared to be more important for metylCbl, where higher levels of holoTC were present for a pattern of daily intake (Fig. 2B). Regarding the pharmaceutical form, 23 subjects used swallowable tablets, 7 chewable/sublingual tablets, 5 drops and 7 different foods considered sources of B12.

Correlation tests were applied between the different elements collected in the study and statistically significant values were obtained (Table IV). In this step, group 3 was eliminated, since many values could not be quantified (frequency of intake, daily intake). An interesting significant correlation appeared between the type of B12 supplement and the duration of veganism (correlation coefficient: -0.342 , $\mathrm{P}=0.044$ ). Older vegans rarely take metylCb, maybe because this form is available on the market only in recent years.

The value of serum holoTC correlated, apart from the type of supplement, with the frequency of administration (frequent administration provided higher values) and with the pharmaceutical form (best with liquid forms, followed by chewable/sublingual and worst with solid ones).

Considering that the body has reserves of vitamin B12 and that the level absorbed is conditioned by the frequency of administration and the amount administered at a time, we made a partial correlation between the value of holoTC and the type of supplement administered, correcting for the duration of a vegan diet, supplementation period with B12, form of administration, frequency of administration and calculated daily dose. The results showed a statistically significant correlation between the type of B12 supplement and the holoTC level, with a correlation coefficient of $-0.39, \mathrm{P}=0.036$.

All variables that were statistically significantly correlated with holoTC values were processed in a linear regression. The dependent variable was the holoTC and the independent variables, the type of supplement, the level of education, the pharmaceutical form and the frequency of intake. The regression was conducted in two ways: i) Enter method, with all factors in one model. This model explains $47 \%$ of the holoTC variability $\left(r^{2}=0.047\right)$. Significant values were observed only for the variable: Type of supplement $(\beta=-0.356, \mathrm{t}=-2.12$, sig.=0.042); ii) Backwards method, in which the algorithm selected 3 variants. The best variant explains $41.5 \%$ of the holoTC variability $\left(r^{2}=0.415\right)$. Significant values were found for the following variables: Type of supplement $(\beta=-0.530$, $\mathrm{t}=-3.88$, sig. $=0.00)$ and frequency of administration $(\beta=0.298$, $\mathrm{t}=2,180$, sig. $=0.00)$ We considered the backwards method as better adapted for exploratory research and referred further to its results.

\section{Discussion}

Currently, more and more people are adopting a plant-based diet. The positive effects on health are highlighted in previous 
Table III. Weekly pattern of B12 supplementation (\% of the participants for each group).

\begin{tabular}{lccccr}
\hline Groups & Once a week (\%) & Twice per week (\%) & Thrice per week (\%) & Daily (\%) & P-value \\
\hline Group 1 & 33 & 28.6 & 4.8 & 33.3 & 0.054 \\
Group 2 & 42.9 & 35.7 & - & 21.4 & \\
\hline
\end{tabular}

Groups: Subjects were divided into 3 categories: Vegans who supplement with cyanCbl (group 1), vegans who supplement with methylCbl (group 2). Group 3 data are not provided here.

Table IV. Significant correlations between parameters of the study (Pearson correlation test).

\begin{tabular}{lcr}
\hline & \multicolumn{2}{c}{ HoloTC level } \\
\cline { 2 - 3 } Study parameters & Correlation coefficient & P-value \\
\hline Type of B12 supplement & -0.572 & $<0.001$ \\
Level of studies & 0.427 & 0.010 \\
Frequency in a week & 0.457 & 0.006 \\
Pharmaceutical form & 0.463 & 0.005 \\
\hline
\end{tabular}

HoloTC, holotranscobalamin.

research (13) but these can only be considered to the extent that the food plans are adequate (14). In the case of vitamin B12, to date, there is no reliable source in the category of unfortified plant foods consumed by humans (15-19). That is why all individuals who consume a plant-based diet are advised to supplement, this group joining other population groups for whom supplementation is recommended. In the absence of supplementation, the consequences are very serious and of an extreme gravity $(20,21)$. Particular age groups are especially exposed to various mineral and vitamin deficiencies, as we have shown in our country $(9,22-25)$, and in the framework of a plant-based diet, the absence of vitamin B12 supplementation can trigger dire consequences (13).

The most available supplements contain cyanCbl and methylCbl. In the present study, we targeted the effectiveness of the two pharmaceutical forms in maintaining a marker of the adequacy of vitamin B12 intake within normal limits, on a group of healthy adults on plant-based diets. HoloTC is considered an appropriate marker, but there are no cut-off points, the values having a general indicative value (26). It is largely considered that normal B12 status is characterized by the following values: Holo-transcobalamin II >45 pmol/1, MMA $<271 \mathrm{nmol} / \mathrm{l}$, and homocysteine $<10 \mu \mathrm{mol} / \mathrm{l}$ (27). Therefore, we considered the holoTC minimal normal value as being $35 \mathrm{pmol} / \mathrm{l}$ (as stated by the analysis reports), evaluating the nutritional status of the group members according to it.

It can be seen that the holoTC values were significantly higher in the group that supplemented using cyanCbl, the association between holoTC values and the type of supplement being maintained even when the statistical analysis controlled for other variables that might influence the B12 level (the duration of consumption of a vegan diet, the period

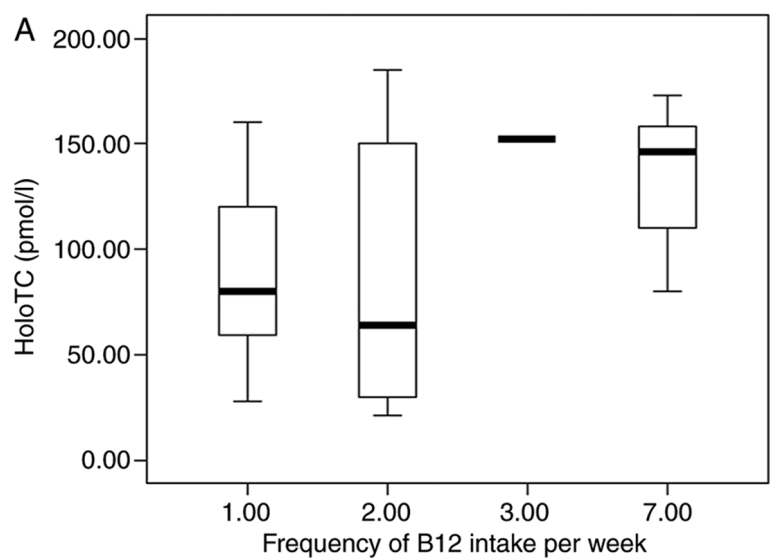

B

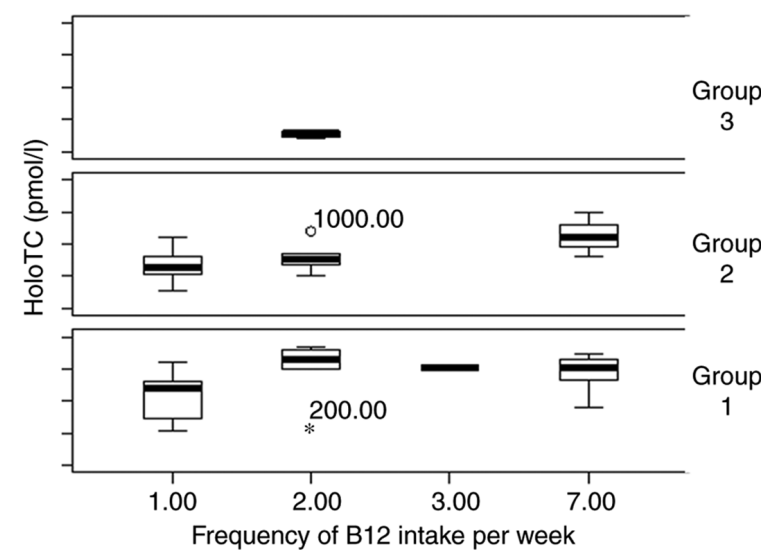

Figure 2. (A) Values of holoTC (pmol/l) in relation to the frequency of the B12 supplement intake. (B) Values of holoTC (pmol/1) in relation to the type of supplement (groups 1-3) and the frequency of intake.

of supplementation with B12, the pharmaceutical form, the frequency of administration and the calculated daily dose).

Regarding this aspect, literature provides contradictory data. One study identified that the percent of absorption of $1 \mathrm{mcg}$ of cyanCbl was $49 \%$, while the identical amount of methylCbl was $44 \%$ (28). In contrast, other studies have shown that cyan $\mathrm{Cbl}$ is excreted 3 times more in the urine than meth$\mathrm{ylCbl}$, which may indicate that methylCbl is better retained in the body (29). There are also studies that showed that differences in bioavailability are insignificant and that, in fact, there are other factors involved (e.g., age or genetics) (7,30). Maybe these factors could also explain that some of the members of group 2 had values below the limit of $35 \mathrm{pmol} / \mathrm{l}$, even though their pattern of supplementation was rather correct. 
In our study, age was not a significant variable, but genetic variations probably left their mark on the results, especially since the group was very small. It should be noted that in both group 1 and group 2 the holoTC values had an important dispersion, which again highlights the influence of individual factors in terms of absorption and use of the vitamin, regardless of the form of the supplement.

Vitamin B12 absorption is an extremely complex process (19) and it is known that the intake of supplements is optimal either in lower and more frequent doses (when absorption is done mainly with the help of intrinsic factor), or in high doses, 1-2 times a week (when absorption with intrinsic factor is completed with diffusion absorption, the rate of which, however, is extremely low) $(31,32)$. In the present case, higher holoTC values were directly correlated with the frequency of supplementation during one week, even when controlling for the level of one time intake. Frequency remains one of the significant predictors of holoTC in regression tests. Other researcher report similar findings, with more frequent supplementation providing better results than one per week high dose (33). That is why the recommendation to opt for a frequent vs. a rarer administration, with an adequate dosage, seems to be an interesting conclusion.

Regarding the recommended doses, while for cyanCbl there are many studies that have indicated the amounts needed to avoid vitamin-induced deficiency in healthy adults or children, for methylCbl most studies have been performed in patients who have either already megaloblastic anemia caused by vitamin 12 deficiency, or there is a neurological impairment (diabetic neuropathy, amyotrophic sclerosis, chemotherapy-induced neuropathy). Thus, the recommended daily amount of cyanCbl for the prevention of deficiency is usually 50-250 mcg/day, while the daily amount of methylCbl that led to good results in neuropathies rose to $1,500 \mathrm{mcg}$ (28). An older study shows that for lower doses, the rate of absorption was equal in both forms but at higher doses cyanCbl was more effective (34). Information on the products of the market is especially confusing in terms of methylCbl. Daily doses vary between 5,000 mcg in one product, $1,000 \mathrm{mcg}$ in another one and $1,250 \mathrm{mcg}$, in a third. In our study, doses varied considerably, mirroring the general confusion regarding the correct level of supplementation. The explanation for the differences in the indicated doses is probably that methylCbl is a less stable compound, which can be subjected to photodecomposition (35). However, an improved communication by the manufacturers of the necessary doses of B12 is compulsory, differentiated on each type of supplement.

Our study showed better holoTC values for liquid or chewable/sublingual supplement forms. There are international confirmations in this regard, related to the onset of 'digestion' of B12 in the oral cavity (36). The problem that remains is that these supplements are more expensive than the classical ones. It should be noted that not all studies confirm the superiority of the forms that use dissolution in the oral cavity (37). New forms, with often-new uses, are currently being developed, and the impact on maintaining normal B12 values will be assessed in the future $(38,39)$.

One interesting but expected finding was that better values of holoTC were correlated with the educational level of the participants; people with higher education naturally adhering to a more adequate plan of supplementation. Again, this indicates a need to better educate on how and when to supplement with vitamins or minerals. Studies conducted in our country have shown a greater concern for nutrition in better-educated population groups $(40,41)$.

A separate group that was excluded from the analysis presented so far was group 3. HoloTC values in this group were substantially lower than in the other two groups. According to the values provided by the laboratory, but also by the values considered normal in general, a value below $35 \mathrm{pmol} / 1$ signifies the lowering of the B12 deposits and the beginning of their depletion. In such conditions, a recommendation to further evaluate the MMA status is useful. In the online environment and in the press different vegetal products are given as sources of vitamin B12, although it is known that they have, at best, inactive structural analogues that often inhibit good functioning by the body of B12 (42-45). Isolated studies contradict these facts, showing that certain products may correct the B12 intake, but confirmation of these results by other reliable research is absent (46). In this regard, better education is needed for people who opt for a plant-based diet, in terms of B12 sources and supplementation adequacy.

Limitation. For reasons related to the financial limitations of this study, only a very small group of individual on a plant-based diet could be considered. We note that the study did not finance the holoTC dosage, but the participants themselves paid for it. The small group did not allow the correction of the results in terms of individual characteristics. We also do not have data on the baseline level of holoTC in each person, but since all of them supplemented for at least 1 year, the importance of the initial level might be not significant. For the moment, we could not follow the study by evaluating the MMA values for persons below $35 \mathrm{pcg} / \mathrm{l}$ of holo TC, as generally recommended.

In conclusion, although plant-based nutrition can be healthy when properly planned, it is almost completely devoid of vitamin B12, which must be provided by supplements. In a group of 42 healthy vegans, the types of cobalamin supplements were statistically significantly related with the holoTC value, with cyan supplements having better performance than methyl ones. As such, we cannot conclude, at least based on the data of the present study, that the administration of meth$\mathrm{ylCbl}$ is superior in maintaining a normal level of holoTC, as a marker of B12 intake, even though some information sources tout it. Also, regardless of total or daily doses, the frequency of administration was an important factor, with more frequent doses providing a better ability to maintain holoTC at higher values. Adequate education is required for adults who opt for a plant-based diet so that any deficiencies due to lack of B12 intake can be avoided.

\section{Acknowledgements}

Not applicable.

\section{Funding}

The research was partially funded by the CORE Program, with the support of the Ministry of Research and Innovation (MCI) (contract 22N/2019, Project PN 190201 02). 


\section{Availability of data and materials}

The datasets used and/or analyzed during the current study are available from the corresponding author on reasonable request.

\section{Authors' contributions}

$\mathrm{CAZ}$ and $\mathrm{RB}$ produced the manuscript in terms of the literature data and the findings of the study. CAZ conducted also the statistical analysis, NB and AM carried out the interviews of the subjects to collect the data. All authors were involved in the conception of the study, interpreted the patient data, and were major contributors in the writing of the manuscript. All authors read and approved the final version of the manuscript to be published.

\section{Ethics approval and consent to participate}

The Ethics Committee of the National Institute of Research and Development for Food Bioresearch, Bucharest, Romania assessed and approved the study. Every participant was informed about the aim of the study, about respecting the privacy during its progress and signed consent to participate. Respondents were informed that data gathered during the study might be published but that personal details would not be included. Signed informed consent included possibility of data publication.

\section{Patient consent for publication}

Not applicable.

\section{Competing interests}

The authors declare that they have no competing interests.

\section{References}

1. Nohr D, Biesalski HK and Back EI: Vitamin B12. In: Encyclopedia of Dairy Sciences. Elsevier, Oxford, pp675-677, 2011.

2. Institute of Medicine (US) Standing Committee on the Scientific Evaluation of Dietary Reference Intakes and its Panel on Folate, Other B Vitamins, and Choline: Dietary Reference Intakes for Thiamin, Riboflavin, Niacin, Vitamin $\mathrm{B}_{6}$, Folate, Vitamin $\mathrm{B}_{12}$ Pantothenic Acid, Biotin, and Choline. National Academies Press (US), Washington, DC, 1998.

3. National Institutes of Health (NIH): Vitamin B12 Fact Sheet for HealthProfessionals.NIH,Bethesda,MD,2020.https://ods.od.nih. gov/factsheets/VitaminB12-HealthProfessional/\#h6. Accessed September 1, 2020.

4. Watanabe F, Yabuta Y, Bito T and Teng F: Vitamin $B_{12}$-containing plant food sources for vegetarians. Nutrients 6: 1861-1873, 2014.

5. Nakos M, Pepelanova I, Beutel S, Krings U, Berger RG and Scheper T: Isolation and analysis of vitamin B12 from plant samples. Food Chem 216: 301-308, 2017.

6. Wolffenbuttel BHR, Wouters HJCM, Heiner-Fokkema MR and van der Klauw MM: The many faces of cobalamin (vitamin $B_{12}$ ) deficiency. Mayo Clin Proc Innov Qual Outcomes 3: 200-214, 2019.

7. Obeid R, Fedosov SN and Nexo E: Cobalamin coenzyme forms are not likely to be superior to cyano- and hydroxyl-cobalamin in prevention or treatment of cobalamin deficiency. Mol Nutr Food Res 59: 1364-1372, 2015.

8. Hvas AM and Nexo E: Holotranscobalamin-a first choice assay for diagnosing early vitamin B deficiency? J Intern Med 257: $289-298,2005$
9. Zugravu C, Rașcu A, Otelea MR and Macri A: Vitamin D from food and supplement intake in pregnancy. A pilot study. Farmacia 68: 150-154, 2020.

10. Nexo E and Hoffmann-Lücke E: Holotranscobalamin, a marker of vitamin B-12 status: Analytical aspects and clinical utility. Am J Clin Nutr 94: 359S-365S, 2011.

11. Fragasso A, Mannarella C, Ciancio A, Scarciolla O, Nuzzolese N, Clemente R, Vitullo E and Sacco A: Holotranscobalamin is a useful marker of vitamin B12 deficiency in alcoholics. ScientificWorldJournal 2012: 128182, 2012.

12. Jarquin Campos A, Risch L, Nydegger U, Wiesner J, Vazquez Van Dyck M, Renz H, Stanga Z and Risch M: Diagnostic accuracy of holotranscobalamin, vitamin B12, methylmalonic acid, and homocysteine in detecting B12 deficiency in a large, mixed patient population. Dis Markers 2020: 7468506, 2020.

13. Mariotti F (ed): Vegetarian and plant-based diets in health and disease prevention. 1st edition. Academic Press, Cambridge, MA, 2017.

14. Craig WJ and Mangels AR; American Dietetic Association: Position of the American Dietetic Association: Vegetarian diets. J Am Diet Assoc 109: 1266-1282, 2009.

15. Rizzo G, Laganà AS, Rapisarda AM, La Ferrera GG, Buscema M, Rossetti P, Nigro A, Muscia V, Valenti G, Sapia F, et al: Vitamin B12 among vegetarians: Status, assessment and supplementation. Nutrients 8: 767, 2016.

16. Haddad EH, Berk LS, Kettering JD, Hubbard RW and Peters WR: Dietary intake and biochemical, hematologic, and immune status of vegans compared with nonvegetarians. Am J Clin Nutr 70 (Suppl 3): 586S-593S, 1999.

17. Guney T, Yikilmaz AS and Dilek I: Epidemiology of vitamin B12 deficiency. IntechOpen Limited, London, 2016. https://www. intechopen.com/books/epidemiology-of-communicable-and-noncommunicable-diseases-attributes-of-lifestyle-and-nature-onhumankind/epidemiology-of-vitamin-b12-deficiency. Accessed November 9, 2016.

18. Gilsing AM, Crowe FL, Lloyd-Wright Z, Sanders TA, Appleby PN, Allen NE and Key TJ: Serum concentrations of vitamin B12 and folate in British male omnivores, vegetarians and vegans: Results from a cross-sectional analysis of the EPIC-Oxford cohort study. Eur J Clin Nutr 64: 933-939, 2010.

19. Obeid R, Heil SG, Verhoeven MMA, van den Heuvel EGHM, de Groot LCPGM and Eussen SJPM: Vitamin B12 intake from animal foods, biomarkers, and health aspects. Front Nutr 6: 93, 2019.

20. Haddad EH, Jaceldo-Siegl K, Oda K and Fraser GE: Associations of circulating methylmalonic acid and vitamin B-12 biomarkers are modified by vegan dietary pattern in adult and elderly participants of the adventist health study 2 calibration study. Curr Dev Nutr 4: nzaa008, 2020.

21. Baroni L, Goggi S, Battaglino R, Berveglieri M, Fasan I, Filippin D, Griffith P, Rizzo G, Tomasini C, Tosatti MA and Battino MA: Vegan nutrition for mothers and children: Practical tools for healthcare providers. Nutrients 11: 5, 2018.

22. Bohiltea RE, Zugravu CA, Neacsu A, Navolan D, Berceanu C, Nemescu D, Bodean O, Turcan T, Baros A and Cirstoiu MM: The prevalence of vitamin D defficiency and its obstetrical effects. A prospective study on Romanian patients. Rev Chim 70: 1228-1233, 2019

23. Bohiltea RE, Zugravu CA, Nemescu D, Turcan N, Paulet FP, Gherghiceanu F, Ducu I and Cirstoiu MM: Impact of obesity on the prognosis of hypertensive disorders in pregnancy. Exp Ther Med 20: 2423-2428, 2020.

24. Iordachescu AC, Cirstoiu MM, Zugravu CA, Teodor OM, Turcan N, Ducu I and Bohiltea RE: Dietary behavior during pregnancy. Exp Ther Med 20: 2460-2464, 2020.

25. Stativa E, Rus AV, Stanescu A, Pennings JS, Parris SR and Wenyika R: Prevalence and predictors of anaemia in Romanian infants 6-23 months old. J Public Health (Oxf) 38: e272-e281, 2016.

26. Green R, Allen LH, Bjørke-Monsen AL, Brito A, Guéant JL, Miller JW, Molloy AM, Nexo E, Stabler S, Toh BH, et al: Vitamin B12 deficiency. Nat Rev Dis Primers 3: 17040, 2017.

27. Herrman W and Giesel J: Vegetarian lifestyle and monitoring of vitamin B-12 status. Clin Chim Acta 326: 47-59, 2002.

28. Gupta JA and Quershi SS: Potential benefits of methylcobalamin: A review. Austin J Pharmacol Ther 3: 1076, 2015.

29. Okuda K, Yashima K, Kitazaki T and Takara I: Intestinal absorption and concurrent chemical changes of methylcobalamin. J Lab Clin Med 81: 557-567, 1973. 
30. Paul C and Brady DM: Comparative bioavailability and utilization of particular forms of B12 supplements with potential to mitigate B12-related genetic polymorphisms. Integr Med (Encinitas) 16: 42-49, 2017.

31. Norris J: Daily needs. Vegan Health, 2020. https://veganhealth. org/daily-needs/\#Vitamin-B12. Accessed September 20, 2020.

32. Berlin H, Berlin R and Brante G: Oral treatment of pernicious anemia with high doses of vitamin B12 without intrinsic factor Acta Med Scand 184: 247-258, 1968.

33. Del Bo' C, Riso P, Gardana C, Brusamolino A, Battezzati A and Ciappellano S: Effect of two different sublingual dosages of vitamin $\mathrm{B} 12$ on cobalamin nutritional status in vegans and vegetarians with a marginal deficiency: A randomized controlled trial. Clin Nutr 38: 575-583, 2019.

34. Adams JF, Ross SK, Mervyn L, Boddy K and King P. Absorption of cyanocobalamin, coenzyme B 12, methylcobalamin, and hydroxocobalamin at different dose levels. Scand J Gastroenterol 6: 249-252, 1971.

35. Juzeniene A and Nizauskaite Z: Photodegradation of cobalamins in aqueous solutions and in human blood. J Photochem Photobiol B 122: 7-14, 2013.

36. Delpre G, Stark P and Niv Y: Sublingual therapy for cobalamin deficiency as an alternative to oral and parenteral cobalamin supplementation. Lancet 354: 740-741, 1999.

37. Sharabi A, Cohen E, Sulkes J and Garty M: Replacement therapy for vitamin B12 deficiency: Comparison between the sublingual and oral route. Br J Clin Pharmacol 56: 635-638, 2003.

38. Siebert AK, Obeid R, Weder S, Awwed HM, Sputtek A, Geisel J and Keller M: Vitamin B-12-fortified toothpaste improves vitamin status in vegans: A 12 -wk randomized placebo-controlled study. Am J Clin Nutr 105: 618-625, 2017.
39. Zant A, Awwad HM, Geisel J, Keller M and Obeid R: Vitamin B12-fortified toothpaste improves vitamin status in elderly people: A randomized, double-blind, placebo-controlled study. Aging Clin Exp Res 31: 1817-1825, 2019.

40. Zugravu CA, Pogurschi EN, Pătrașcu D, Iacob PD and Nicolae CG: Attitudes towards food additives: A pilot study. Ann Univ Dunarea de Jos Galati Fascicle VI Food Technol 41: 50-61, 2017.

41. Zugravu CA: Eating habits and influential factors for mothers and children in Romania. Int J Collab Res Inter Med Public Health 4: 362-374, 2012.

42. Norris J: Vitamin B12 in plant foods. Vegan Health, 2020. http://veganhealth.org/vitamin-b12-plant-foods/. Accessed September 21, 2020.

43. Watanabe F, Katsura H, Takenaka S, Fujita T, Abe K, Tamura Y, Nakatsuka T and Nakano Y: Pseudovitamin B(12) is the predominant cobamide of an algal health food, spirulina tablets. J Agric Food Chem 47: 4736-4741, 1999.

44. Donaldson MS: Metabolic vitamin B12 status on a mostly raw vegan diet with follow-up using tablets, nutritional yeast, or probiotic supplements. Ann Nutr Metab 44: 229-234, 2000.

45. Watanabe F, Miyamoto E, Fujita T, Tanioka Y and Nakano Y: Characterization of a corrinoid compound in the edible (blue-green) alga, Suizenji-nori. Biosc Biotechnol Biochem 70: 3066-3068, 2006.

46. Merchant RE, Phillips TW and Udani J: Nutritional supplementation with chlorella pyrenoidosa lowers serum methylmalonic acid in vegans and vegetarians with a suspected vitamin B12 deficiency. J Med Food 18: 1357-1362, 2015. 